\title{
Les changements d'occupation des sols dans la Béqaa Ouest (Liban): le rôle des actions anthropiques
}

\author{
Hussein El Hage Hassan ${ }^{1, *}$, Françoise Ardillier-Carras ${ }^{2}$ et Laurence Charbel $^{3}$ \\ ${ }^{1}$ Laboratoire de recherche CEDETE (EA 1210), Département de géographie, Université Libanaise, Beyrouth, Liban \\ ${ }^{2}$ Laboratoire de recherche CEDETE (EA 1210), Département de géographie, Université d'Orléans, Orléans, France \\ 3 Département de géographie, Université Libanaise, Fanar, Liban
}

\begin{abstract}
Résumé - L'évolution des modes d'occupation du sol porte l'empreinte des activités anthropiques et correspond à un processus dynamique assez complexe, qui agit sur les ressources naturelles. La région agricole de la Béqaa Ouest est caractérisée par des conditions naturelles favorables pour une production agricole diversifiée. La télédétection montre l'ampleur des changements des modes d'occupation du sol entre 1962 et 2019. L'analyse diachronique révèle une régression du couvert végétal au profit des tissus urbains dans la plaine. L'interprétation des résultats doit tenir compte des causes locales de cette mutation en mettant en évidence les pratiques culturales et pastorales, les facteurs économiques, l'extension urbaine, les flux migratoires syriens et le déboisement qui fragilise le milieu en provoquant le décapage des couches superficielles. L'indice de brillance de l'image Ikonos de haute résolution indique la sévérité de cette dégradation. Tout cela interroge l'avenir agricole de cette région dont les productions constituent un apport essentiel aux marchés nationaux.
\end{abstract}

Mots clés : Béqaa / couvert végétal / image satellitaire / érosion / agriculture

\begin{abstract}
Land use changes in West Beqaa (Lebanon): role of the anthropogenic activities. The evolution of land tenure patterns reflects the footprint of anthropogenic activities and is rather a complex dynamic process that acts on natural resources. The agricultural region of the West Beqaa is characterized by favorable natural conditions for diversified agricultural production. Remote sensing shows the extent of changes in land use between 1962 and 2019. Diachronic analysis reveals a regression of the vegetation cover in favor of urban fabric in the plain. The interpretation of the results must take into consideration the local causes of this mutation by highlighting cultural and pastoral practices, economic factors, urban extension, Syrian migratory flows, and deforestation that weakens the environment through stripping away the superficial layers. The brightness index of the high-resolution Ikonos image was used to determine the severity degree of degradation. All this questions the agricultural future of the region, whose production is an essential contribution to the national market.
\end{abstract}

Keywords: Beqaa / vegetation cover / satellite image / erosion / agriculture

\section{Introduction}

Situé sur la marge orientale du bassin méditerranéen, dans la Béqaa Ouest, le terrain de cette étude est caractérisé par des mutations de l'occupation du sol qui s'inscrivent dans une évolution conjoncturelle du Liban, notamment politique, économique, et démographique amplifiée depuis 2010 par l'immigration liée au conflit syrien, pays limitrophe de la

\footnotetext{
*Auteur de correspondance :

hussein.el-hage-hassan@ul.edu.lb
}

Béqaa. Le suivi des modifications du mode d'occupation et de l'utilisation du sol doit être une priorité pour les décideurs politiques, pour la planification régionale comme pour l'étude et la compréhension de l'environnement (Foody, 2002). La situation est alarmante, certains secteurs déboisés deviennent des zones de ruptures écologiques.

Ces changements d'occupation du sol modifient aussi les paysages jusqu'à perturber leur diversité, l'un des points forts de la Béqaa Ouest. Le développement des techniques de télédétection et des systèmes d'information géographique permet d'apprécier les transformations du couvert du sol. La disponibilité d'une série de données, multi-dates, 1962 et 2019, 


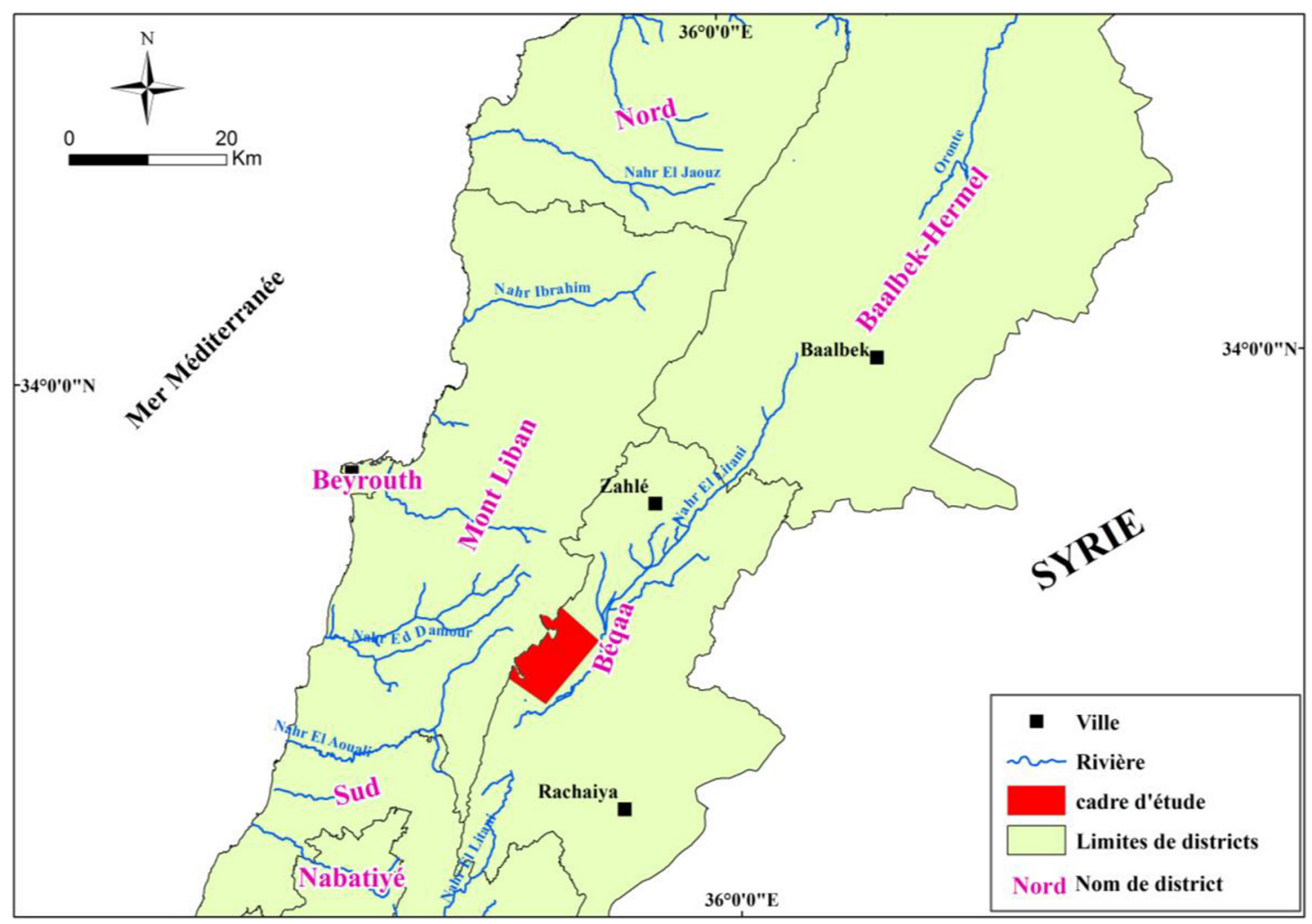

Fig. 1. Localisation du cadre d'étude.

Fig. 1. The study area.

au 1:200000, avec prise de vues en fin d'été, a permis de cartographier les mutations avérées du couvert végétal. Pour relier l'occupation du sol aux usages concernés, ce que nous appelons l'utilisation du sol, nous nous sommes également appuyés sur des enquêtes de terrain et des entretiens auprès d'exploitants agricoles (dans la Béqaa Ouest), de coopératives (à l'échelle nationale) et de la population résidante. La dynamique paysagère sera mise en évidence par une approche diachronique qui mettra l'accent sur les processus anthropiques à l'œuvre.

Après la présentation du cadre d'étude, nous décrirons la méthodologie utilisée pour cette approche spatiale. Puis, nous présenterons les résultats. Et enfin, nous proposerons des conclusions.

\section{Cadre d'étude}

La Béqaa Ouest, un espace agricole actif et servi par des conditions naturelles favorables, est intéressante pour étudier l'évolution du mode d'occupation du sol au Liban. Le choix s'est porté sur un secteur situé au cœur de la Béqaa ouest, entre $33^{\circ} 44^{\prime}-33^{\circ} 37^{\prime}$ de latitude Nord et $35^{\circ} 41^{\prime}-35^{\circ} 49^{\prime}$ de longitude Est (Fig. 1). Le terrain d'étude, représentatif de l'évolution observée dans la plus grande partie de la Béqaa, occupe 6437 ha; nous y disposons d'informations importantes dans la continuité de nos travaux de recherche et de thèse conduits depuis 2004 (El Hage Hassan, 2011).

Le secteur, qui est drainé vers le sud par le Litani, associe deux unités morphologiques :
- les versants orientaux du Mont Liban, dont le point culminant atteint $1920 \mathrm{~m}$, sont particulièrement sensibles aux phénomènes érosifs. Cet ensemble se distingue par une forte valeur de pente $\left(52^{\circ}\right)$, un sol à faible épaisseur et un couvert végétal clair. L'érosion est particulièrement active dans ce secteur en raison de l'absence d'un couvert végétal protecteur;

- la plaine, située à l'Est, qui s'ouvre à $870 \mathrm{~m}$ d'altitude, est caractérisée par un sol profond et une agriculture diversifiée. Elle est traversée par le Litani, fleuve permanent qui parcourt plusieurs centaines de kilomètres avant de se jeter dans la Méditerranée et constitue une source d'eau importante pour l'irrigation durant la période sèche estivale.

Le substratum géologique des versants est composé de roches du Jurassique moyen, affleurant à certains endroits et présentant deux faciès : le calcaire dolomitique et le calcaire massif. La plaine est dominée par les dépôts du quaternaire, le calcaire marneux et le calcaire dolomitique du Cénomanien (C4), les marnes et le calcaire marneux Miocène lacustre, et le calcaire du Crétacé inférieur.

Les propriétés des différents types de sols (teneur en matière organique, profondeur...) présents dans le secteur d'étude jouent sur leurs aptitudes culturales (FAO, 1989). Seul le leptosol lithique, qui domine sur les versants, ne permet aucune culture et ne tolère qu'une végétation clairsemée ou forestière (El Hage Hassan et al., 2016). Ce sol, peu profond, 
est caractérisé par une faible capacité à la rétention d'eau et il est facilement érodé.

Le climat, de type méditerranéen, se caractérise par la répartition saisonnière des précipitations, leur intensité et la longueur de la période sèche (mai à septembre) suivie d'une période pluvieuse (novembre à avril). Le secteur est situé entre les isohyètes 700 et $1000 \mathrm{~mm}$. Le nombre de jours de pluie est de 120/an en moyenne.

Les populations locales libanaises connaissent une croissance régulière. Mais la région est caractérisée par une faible densité (124 personnes $/ \mathrm{km}^{2}$ ) qui s'explique en partie par la diminution des rendements agricoles, l'exode rural, l'absence de service administratif et la topographie mouvementée. Malgré la faible densité, le couvert végétal des versants ne cesse de se dégrader sous l'impact des activités humaines et de la transhumance saisonnière, qui ont entraîné des modifications de l'état du sol. Le surpâturage des brebis a généré un processus de déforestation qui a accéléré par la suite l'érosion en montagne. Ceci a conduit au développement des terrains incultes.

Avec des nuances issues de la configuration physique, la région d'étude présente une mosaïque de parcelles agricoles. Dans la plaine, ce sont les cultures qui s'imposent alors que sur les versants (ouest), le sol est plutôt occupé par des surfaces boisées dégradées. L'agriculture constitue la principale ressource pour la population active agricole, avec 624 exploitations (El Hage Hassan, 2011). Dans la plaine, on pratique les cultures céréalières et maraîchères, ainsi que l'arboriculture fruitière et la viticulture. L'élevage est une activité complémentaire pour les agriculteurs, avec la production de foin et des pâturages naturels permanents pour l'alimentation du bétail (bovin, ovin et caprin). La montagne est historiquement exposée au déboisement pour assurer les besoins de la population en bois de chauffage et en charbon de bois. Aujourd'hui, la régression des surfaces boisées et des broussailles demeure nette. Le déboisement est, entre autres, à l'origine d'un processus érosif sur des sols de versants fragiles (El Hage Hassan et al., 2018).

Afin de comprendre l'érosion et l'évolution de l'occupation du sol, nous avons mis en œuvre une méthodologie visant à mettre en évidence les processus tant physiques que socioéconomiques actuels.

\section{Méthodologie appliquée à l'étude des changements d'occupation du sol dans la Béqaa Ouest et matériel d'étude}

C'est d'abord par la cartographie que l'étude des mutations actuelles d'occupation du sol est abordée. Les photos aériennes et les images satellitaires offrent une vision particulière de la surface terrestre, adaptée à la compréhension de grandes étendues et à l'interprétation de paysages parfois complexes. Les techniques de télédétection permettent d'apprécier les transformations du couvert du sol et d'élaborer des analyses diachroniques à l'aide de traitements numériques.

Afin d'examiner les mutations du couvert du sol, nous nous sommes basés sur la méthode de comparaison des classifications des cartes topographiques et des images satellitaires acquises à des dates différentes. Le principe consiste à assigner des classes aux documents précités avant de détecter les changements. Cette méthode fournit des informations détaillées et permet de produire la carte des changements (Sako et al., 2019).

L'étude du changement portera sur les différents types d'usage : agricole, boisé, et urbain. Le suivi est effectué sur les périodes 1962, 1998, 2013 et 2019.

Pour analyser l'évolution de l'occupation du sol, il a été nécessaire de:

- disposer de données (photos aériennes, cartes topographiques, images satellitaires) suffisantes pour couvrir le terrain d'étude;

- employer deux images satellitaires à l'échelle, du 1/20 000, de type Landsat 1998 (résolution cinq mètres) et Ikonos 2013 (résolution d'un mètre), permettant de cartographier avec précision les différentes unités de la couverture du sol;

- employer une image satellitaire (Google Earth, 2019), permettant de déterminer l'amplification du mitage résidentiel due à l'afflux des réfugiés syriens ;

- intégrer les données cartographiques dans un SIG, ce qui nécessite de les géoréférencer (projection conique conforme de Lambert) et de les caler (système de coordonnées de référence de la zone) pour rendre comparables les couches de 1962, 1998, 2013 et 2019;

- cartographier la couverture du sol, à partir de la vectorisation des cartes topographiques, et établir une classification dirigée qui se réfère à des zones témoins basées sur le positionnement de réalités-terrain;

- produire une carte synthétique de changement, à partir de la combinaison des cartes thématiques précédentes.

Il est difficile de préciser la contribution des facteurs climatiques et de la composante anthropique dans le processus de changement du couvert végétal. Le contexte interne, propre à la situation économique et politique du Liban, intervient aussi dans ce sens. L'analyse diachronique de différentes données cartographiques et d'images demeure notre source principale d'information pour observer les changements d'usage du sol. Cette analyse a été complétée par des missions saisonnières de terrain et par des entretiens visant à appréhender la taille des exploitations, la perception de l'évolution des ressources, l'historique d'exploitation des surfaces boisées et les effets de l'anthropisation sur l'environnement.

Pour diriger la présente étude, nous avons consulté les documents suivants :

- la carte des sols de Rachaya au 1/50000, publiée par le Conseil national de la recherche scientifique du Liban (CNRSL);

- les cartes topographiques de Saghbine, Joubb Jannin et Ghazzé au 1/20 000 (courbes de niveau à $10 \mathrm{~m}$ ) publiées en format raster par la Direction des affaires géographiques de l'armée libanaise (DAGG);

- une image satellite Ikonos à l'échelle $1 / 20000$, prise en août 2013, pour définir le mode d'occupation du sol;

- une image Landsat 1998 à l'échelle du 1/20 000, qui nous a été fournie vectorisée par le CNRS libanais;

- la carte géologique de Rachaya au 1/50 000 (Dubertret et Wetzel, 1951);

- les données statistiques issues du CNRS libanais en 2010. 


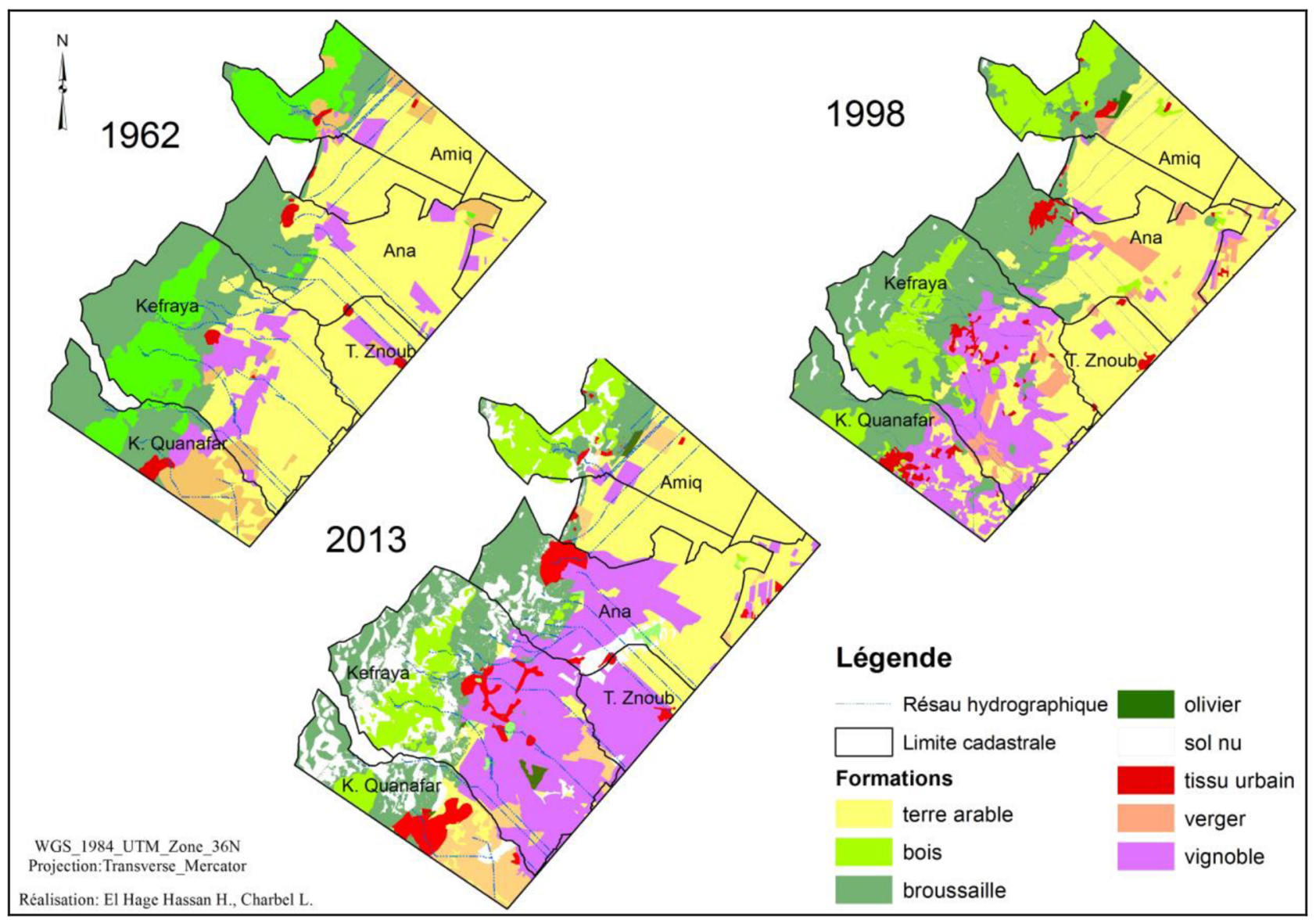

Fig. 2. Mode d'occupation du sol en 1962, 1998 et 2013.

Fig. 2. Land use in 1962, 1998 and 2013.

\subsection{Exploitation des données numériques}

Les données sont d'abord exploitées à partir des modes d'occupation du sol en 1962, puis par une étude comparative avec les données de 2008, 2013 et 2019.

\subsubsection{Mode d'occupation du sol en 1962 et 1998}

Les photos aériennes ont permis d'acquérir des informations sur le mode d'occupation du sol de la région d'étude. Ces photos, prises en 1962, numérisées en utilisant un scanner de photogrammétrie de haute résolution, ont permis à la DAGG de produire les cartes topographiques à l'échelle 1/20000. L'interprétation des cartes topographiques a consisté à vectoriser les différentes unités d'occupations du sol. Ces entités créées sous forme d'unités polygonales reflètent l'image de l'occupation du sol. La discrimination porte sur le tissu urbain et les différentes formations végétales. Six unités d'occupation du sol ont été retenues (Fig. 2) : terres arables, vergers, vignobles, bois, tissu urbain et broussailles. Pour les données de 1998, la démarche précédente de vectorisation a été adoptée par le CNRSL. Sept unités ont été définies : terres arables, vergers, vignobles, bois, tissu urbain, broussailles et oliviers.

\subsubsection{Mode d'occupation du sol en 2013 et 2019}

L'interprétation des images satellitaires, de haute résolution, met en évidence la répartition des différents éléments du couvert du sol. En vue d'une analyse des mutations de l'occupation du sol, plusieurs méthodes de télédétection ont été utilisées (Andrieu et Mering, 2008).

Pour rendre comparables les images satellitaires (Ikonos et Landsat), nous avons suivi la démarche de vectorisation adoptée pour les cartes topographiques. Certaines classes de références ont été sélectionnées sur l'image satellitaire et validées par des relevés de terrain et le GPS. Pour certains endroits inaccessibles, nous avons eu recours à la classification par maximum de vraisemblance pour confirmer la classe des surfaces boisées par rapport aux surfaces incultes. Il a été nécessaire de définir les caractères des deux unités en amont (Skupinski et al., 2009). L'interprétation des images de haute résolution met en évidence la répartition des différents types d'occupation et permet de cartographier les éléments du couvert du sol. Les classes de référence ont été sélectionnées sur l'image satellitaire et validées par des relevés de terrain et le GPS. Cette démarche peut engendrer une discrimination à l'image. Pour la suite, les images produites ont été soumises à des techniques de posttraitement d'image (corrections, mosaïques d'images et améliorations). Un regroupement de huit classes thématiques a été réalisé. Ce regroupement a favorisé la précision spatiale des classes, en élargissant leur portée sémantique (Lagabrielle et al., 2007). À l'issue de la classification, huit unités d'occupation de sol ont été retenues (Fig. 3): terres 


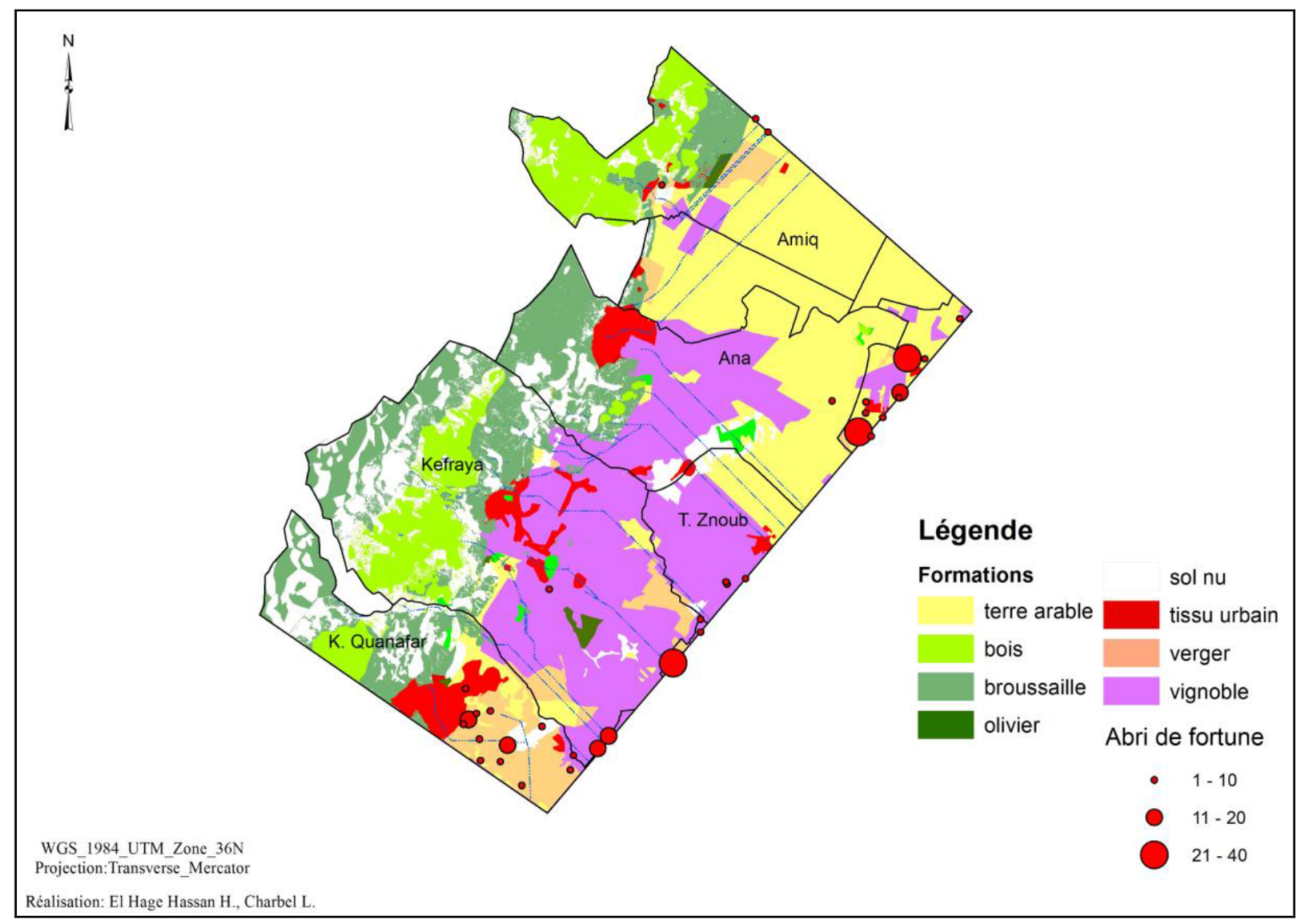

Fig. 3. Mode d'occupation du sol en 2019.

Fig. 3. Land use in 2019.

arables, vergers, vignobles, bois, tissu urbain, broussailles et oliviers. Cependant, pour la carte de 2019, nous avons subdivisé le tissu urbain entre bâtiments et abris de fortune, afin de déterminer l'impact de l'immigration syrienne.

\section{Analyse des changements spatio- temporels de l'occupation du sol}

Le croisement spatial des cartes a conduit à la production de la carte des changements. L'analyse spatio-temporelle des données confirme l'évolution des différentes unités.

La démarche entreprise mène à moduler le tableau 1 des pertes et des gains et la figure 3 , qui permettent de confronter les différentes unités entre 1962 et 2019. Mais, lorsque l'on examine ces données, la situation apparaît diversifiée, l'évolution au sein des unités variant d'un secteur à l'autre. De façon générale, la régression du couvert végétal est remarquable, à l'exception des surfaces cultivées en vignobles et en oliveraies qui ont connu une expansion. Par ailleurs, la carte des changements indique qu'au cours de la période 1962 2019, le paysage de la région a été modifié. En effet, les surfaces boisées et les broussailles ont été fragmentées. Les terrains incultes ont gagné 1256 ha. Les espaces artificialisés se sont étendus.

\subsection{Principaux changements d'usage du sol entre 1962 et 2019}

L'étude des modifications d'usage du sol portera sur huit grands types: les terres arables, les surfaces boisées et les broussailles, les surfaces artificialisées, les vergers, les vignobles, les oliviers et les terrains incultes (Fig. 4).

\subsubsection{Les terres arables (céréales, maraîchage) représentent l'unité agricole la plus importante dans la plaine de la Béqaa}

Cette utilisation du sol, qui domine une large partie de la plaine, se trouve aussi sur les contreforts en petites surfaces. Elle occupe des sols argileux, argilo-sableux et argilo-limonosableux. Les sols concernés sont profonds, alcalins, avec un pourcentage de cailloux en surface inférieur à $5 \%$.

Le choix entre les céréales et les cultures maraîchères dépend, selon les observations de terrain, de la disponibilité des ressources en eau et de la demande du marché. La superficie exacte de chacune des cultures reste variable, compte tenu des facteurs précités. Sur la période considérée (entre 1962 et 2019), la régression des terres arables est estimée à 622 hectares. Ces terres ont été converties généralement en vignoble dans la plaine et en surface urbanisée sur les contreforts. 
Tableau 1. Les différentes composantes de l'occupation du sol entre 1962 et 2019.

Table 1. The different components of land use between 1962 and 2019.

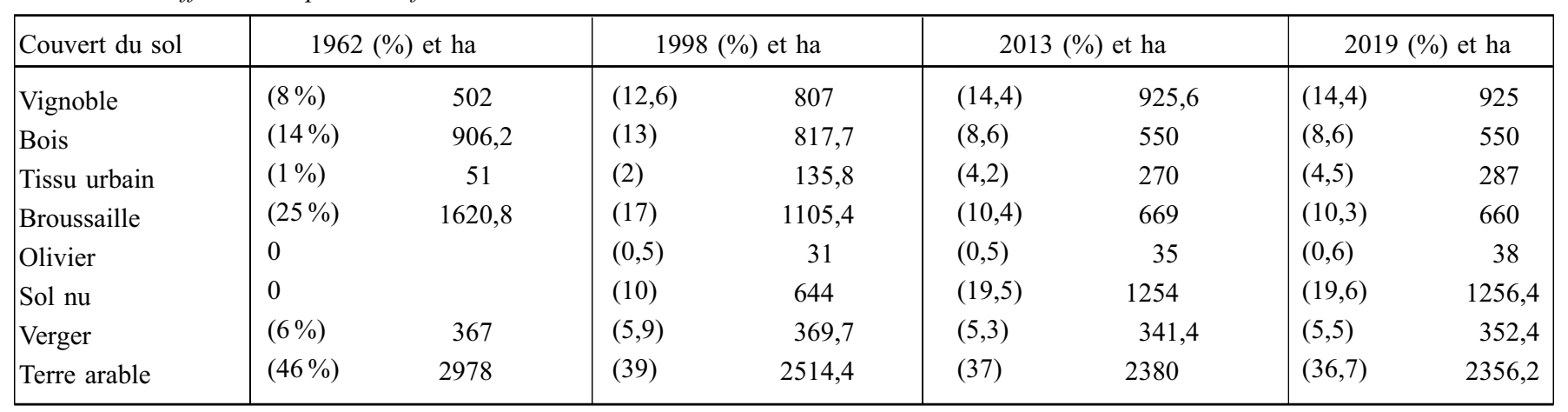

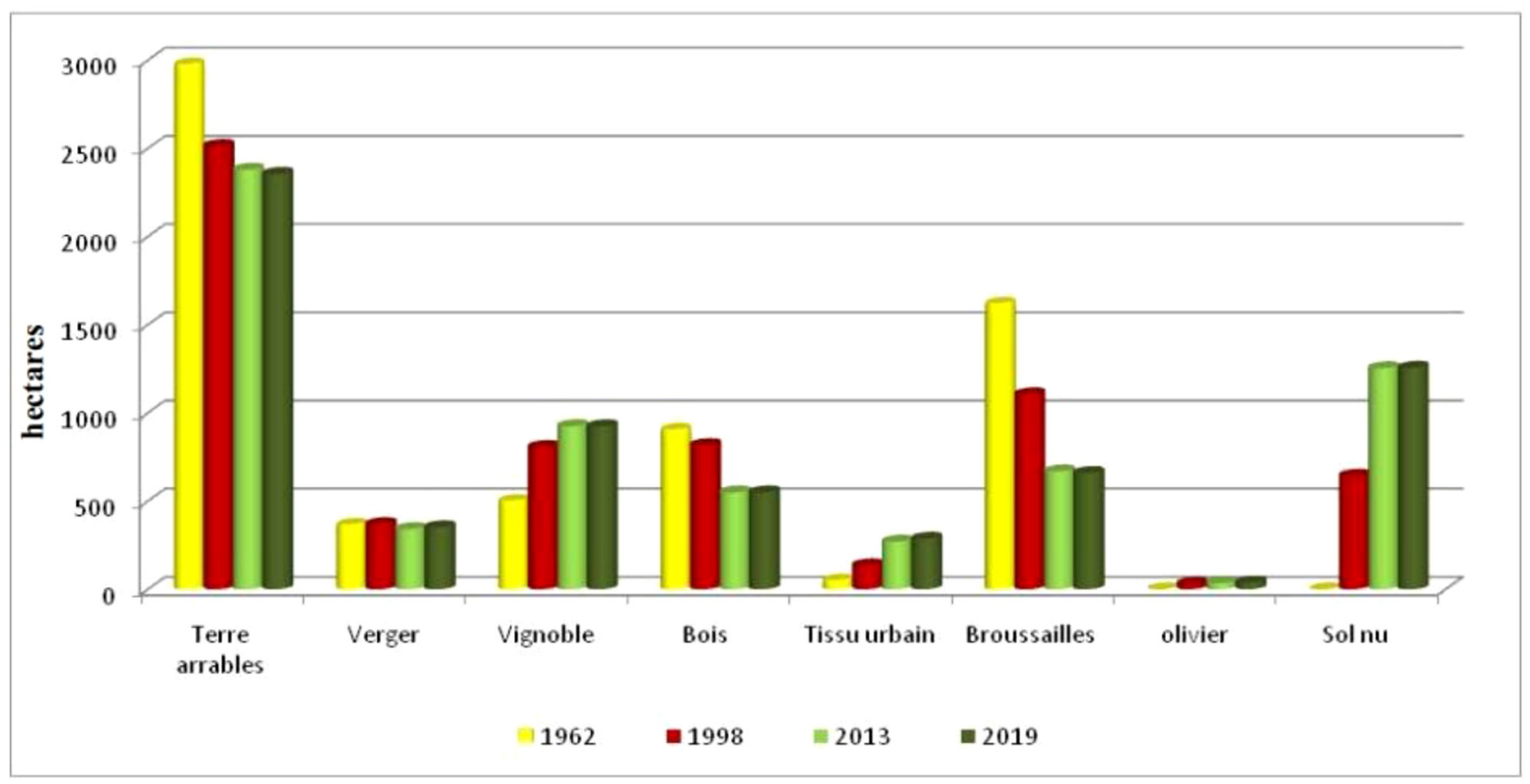

Fig. 4. Répartition des différents éléments d'occupation du sol entre 1962 et 2019.

Fig. 4. Distribution of different elements of land use between 1962 and 2019.

\subsubsection{L'extension des surfaces urbanisées a entraîné une forte pression sur les terres cultivables}

L'examen de la carte des changements montre une progression importante de l'unité artificialisée aux dépens des unités agricoles, liée à l'accroissement de la population locale. On passe de 51 ha en 1962, à 136 en 1998, à 270 en 2013, pour atteindre 287 ha en 2019. Malgré la densification du tissu urbain existant, l'analyse des cartes indique l'étalement de ces structures. L'artificialisation a ainsi touché des sols agricoles éloignés des centres urbains. Cette extension spatiale désordonnée, qui est un défi pour l'aménagement du territoire, s'opère majoritairement au détriment des terres arables et des cultures pérennes. De plus, de nouvelles concurrences sont apparues : depuis 2011, début des flux migratoires syriens causés par la guerre, le travail de terrain montre de nouvelles habitations installées dans 350 abris de fortune, sur des secteurs au potentiel productif, qui font peser un risque sur l'activité agricole et transforment le paysage. De nombreux exploitants propriétaires alimentent ce phénomène en espérant réaliser plus de bénéfices en louant les terrains agricoles à des réfugiés (Fig. 3).

\subsubsection{Les oliviers représentent l'une des cultures les plus importantes au Liban}

Cette formation arborée, qui a fait son apparition depuis moins de 20 ans, est l'unité agricole la moins importante, tant pour la surface ( $38 \mathrm{ha}$ ) que pour la production. En effet, l'augmentation des surfaces d'oliveraies, sur les sols pierreux, difficiles à travailler, est associée à la diminution des terres arables et des broussailles sur les collines. Les oliviers, qui sont résistants à la sécheresse, participent à la fixation des sols sur les versants, offrant ainsi une protection contre l'érosion hydrique. 


\subsubsection{Les vignobles restent une des plus anciennes cultures en place}

Les vignes sont cultivées principalement pour la production du raisin de cuve (Merlot). En 2019, les surfaces viticoles s'étendent sur 925 ha. La majorité se trouve dans la plaine, sur des sols argileux, limoneux et argilo-sableux, profonds, avec un pourcentage de cailloux en surface qui varie entre 5 et $40 \%$. Par ailleurs, les cartes multi-dates indiquent aussi la présence de la vigne sur les collines, sur un sol argilo-limono-sableux, avec un pourcentage de cailloux en surface qui varie de 40 à $80 \%$.

La viticulture a conquis, depuis 1962, 423 ha au détriment des terres arables, doublant ainsi sa surface.

\subsubsection{Les vergers d'arbres fruitiers sont bien entretenus afin de maximiser la production}

Les arbres fruitiers à pépins ou à noyaux et à feuilles caduques (poiriers, pêchers et amandiers) sont les principales productions fruitières. Ces cultures s'étalent sur des sols argileux, peu caillouteux, profonds, dans la plaine et sur des sols argilo-limono-sableux, très caillouteux et moyennement profonds sur les contreforts. D'une façon générale, les vergers sont quasiment restés stables en surface (367 ha en 1962 et 352 ha en 2019), avec une faible perte de quelques hectares au niveau des contreforts.

\subsubsection{Les surfaces boisées et les broussailles représentent les plus forts changements intervenus depuis 1962}

Il s'agit de formations arbustives et buissonnantes qui dominent les versants ouest du Mont Liban. Ces formations végétales, qui s'étendent sur un sol sableux très caillouteux et peu profond, ont été dissociées et fragmentées. En 1962, ces surfaces occupaient 2527 ha. La carte de 1998 montre une régression de 604 ha. En 2019, ces formations ne couvrent que 1210 ha. La destruction des surfaces boisées conduisant au développement des terrains incultes et à l'affleurement de la roche nue, façonne le paysage de cette région. Les besoins de nouveaux espaces pour la production alimentaire ont poussé les paysans à convertir une partie des broussailles en cultures céréalières et en vergers.

\subsubsection{Les terrains incultes pauvres en végétation progressent}

Il s'agit de plaques de sols mis à nu qui occupent une large portion des versants et se trouvent insérés dans les forêts dégradées. En 1998, les terrains incultes couvraient 644 ha. En 2019 , ce chiffre est estimé à 1256 ha, soit $19,7 \%$ de la superficie totale de la région d'étude. Cette progression, au détriment des bois et des broussailles, résulte de l'action de l'homme. En effet, les pratiques de surpâturage ont marqué largement ces secteurs. L'élevage demeure une activité traditionnelle de cette région. L'exploitation commerciale du bois (chauffage et usage domestique) et la dégradation des sols, combinées à l'abandon de ces surfaces, ont amplifié l'extension des terrains incultes.

\section{Facteurs explicatifs de l'évolution de l'utilisation du sol}

L'évolution du mode d'occupation du sol invite à une réflexion sur les processus socio-économiques et environnementaux qui en sont la cause. Les changements d'usage des sols reflètent les stratégies d'acteurs multiples qui se situent à différentes échelles (Liban, Béqaa, secteur d'étude). La guerre, les marchés agricoles, les stratégies foncières, mais aussi leurs effets sur la fertilité des sols engendrent des situations nouvelles qui agissent sur le couvert végétal. Les exploitants agricoles intègrent l'ensemble de ces variables pour les choix de culture, afin de répondre à leurs objectifs économiques (Houet, 2006). L'évolution constatée relève d'une complexité temporelle qui combine le facteur anthropique et le milieu physique (Grégoire et Litaudon-Jouve, 2004). Afin d'appréhender cette complexité, il était nécessaire d'analyser les transformations de l'occupation du sol en fonction de facteurs socio-économiques pouvant être à l'origine des évolutions observées.

Dans notre cas d'étude, un des principaux facteurs de déstabilisation de l'occupation du sol tient à la géopolitique régionale. L'instabilité du Liban et du Moyen Orient, avec la guerre civile de 1975 à 1990, a entraîné l'abandon de terres agricoles. En 1982, la surface abandonnée a été estimée à 215000 ha dans la Béqaa ouest (Hamze, 1991). De même, la vulnérabilité de la Syrie et de sa situation politique et l'insécurité des voies de communication ont réduit considérablement les échanges internationaux et par conséquence la surface agricole.

\subsection{Le déboisement et ses conséquences sur le milieu}

Les surfaces boisées et les broussailles interviennent dans l'écosystème et jouent un rôle pour la protection du sol contre l'érosion hydrique. La région d'étude, notamment sur les versants abrupts, est confrontée à un risque de dégradation, lié au déboisement (chêne, genévrier...).

Pour traiter la fragmentation du paysage forestier et de sa perception, plusieurs actions doivent être évoquées à l'échelle nationale et locale. La loi 558 portant sur la protection des forêts au Liban et l'interdiction d'y exercer toute activité a été mise en vigueur en 1996. L'absence d'un cadre juridique qui impose la protection de ces surfaces avant 1996 a mené à la diminution de certaines espèces végétales. Des observations précises, à Amiq, Ana et Kefraya, de 2011 à aujourd'hui, ont permis d'identifier des plages affectées par l'érosion aréolaire (El Hage Hassan et al., 2013). L'effet conjugué de certains facteurs liés aux éléments naturels (précipitations, sol, pentes) et des activités humaines telles que le surpâturage et l'exploitation incontrôlée du bois, au cours de la guerre civile, pour en faire de l'énergie, expliquent la dynamique érosive en l'absence d'une politique de restauration (Dan Wenhong, 2011).

\subsection{L'influence de la situation des marchés}

L'ouverture de l'économie agricole vers les marchés internationaux influence l'évolution du couvert végétal au 
Liban. L'exigence commerciale en productions agricoles spécifiques (fruits) génère des contraintes et des concurrences qui n'épargnent pas les régions rurales. Pour les marchés extérieurs, il s'agit surtout des pays à bas revenus comme la Syrie (avant la guerre) où le coût de la main-d'œuvre est beaucoup moins élevé. De plus, le coût élevé des semences implique l'augmentation des prix des produits libanais destinés à l'export, causant la mévente et l'affaiblissement des revenus pour les agriculteurs qui, les années suivantes, sont contraints à changer de cultures, pour limiter les pertes.

Dans les années 1990, le raisin de table a traversé une crise liée à l'accroissement de la concurrence. Face à cette situation, les viticulteurs ont procédé à l'arrachage des vignes en vue d'opérer une reconversion qualitative, à l'image de ce qui s'est opéré dans d'autres vignobles méditerranéens (Arnal et al., 2013). En revanche, les vignobles de raisins de cuve ont souvent été maintenus et renouvelés. Cela est dû à l'implantation de producteurs de vin reconnus, comme Château Kefraya (en 1946) dans la région d'étude. Ces entreprises viti-vinicoles sont un atout, pour le marché libanais et pour l'exportation, contribuant ainsi au maintien de ce couvert végétal.

\subsection{Le rôle de l'urbanisation dans les changements d'occupation du sol}

L'évolution du tissu urbain est soumise à la loi sur l'urbanisme, qui exige l'élaboration de plans et de règlements d'urbanisme locaux, à l'exception des circonscriptions rurales où les plans sont facultatifs. Cependant, l'absence de plan d'urbanisme impose aux circonscriptions rurales de se soumettre au code national de la construction. Malgré le rôle des autorités municipales dans l'application de la législation, il semble difficile de faire respecter ce code. L'extension urbaine, qui est menée de manière confuse, s'opère principalement autour des noyaux villageois. Le mitage urbain y est peu apparent.

\subsection{Rôle du régime foncier et de l'irrigation : facteurs de transformations paysagères dans le secteur agricole}

En 1961, le recensement général fait état de 143000 exploitations au Liban. En 1999, ce nombre atteignait 195829 (Ministère de l'Agriculture, 1999). Les exploitations de moins d'un hectare représentent $20 \%$ de la surface agricole, alors que les exploitations de plus de 10 ha pèsent $30 \%$ du nombre total (Ministère de l'Agriculture, 2008).

Aujourd'hui, l'entrée de nouveaux acteurs et le partage entre les descendants ont multiplié le nombre d'exploitations (Tab. 2). Le régime foncier intervient dans les mutations du mode d'occupation du sol. Bien que la législation libanaise ait définit les différents statuts fonciers (moschaa: propriété communale et publique; mulk: propriété privée) et les modalités d'attribution des droits d'utilisation du foncier, les lois qui régissent les propriétés publiques et communales sont parfois négligées. Les domaines publics, exploités sans permission par les villageois, peuvent être partagés entre les héritiers malgré l'absence d'inscription légale d'un contrat dans le registre foncier. Cependant, les modalités d'exploita-
Tableau 2. Nombre d'exploitations agricoles dans la région d'étude. Table 2. Number of farms in the study area.

\begin{tabular}{|l|c|}
\hline Villages & Nombre d'exploitations \\
\hline Ch. Ammiq & 13 \\
Ana & 131 \\
D. Tahniche & 6 \\
Tall Znoub & 101 \\
Kefraya & 88 \\
K. Qanafar & 179 \\
Ammiq & 106 \\
\hline
\end{tabular}

tions adaptées par les résidants sur les propriétés privées sont appliquées aux propriétés publiques et communales.

Dans la plaine, la majorité des exploitations agricoles sont des propriétés privées et les mutations de ces surfaces dépendent des modes de faire-valoir. Pour les surfaces agricoles en faire-valoir direct, les exploitants propriétaires privilégient les productions arboricoles fruitières. En revanche, sur les surfaces agricoles en location, les exploitants pratiquent plutôt des cultures annuelles. Ceci signifie que la rotation des cultures est plus importante pour les terres en fermage que pour celles en propriété.

Globalement, les versants et les contreforts, occupés par les surfaces boisées et les pâtures, relèvent du domaine communal qui tolère le pâturage. En effet, leur moindre qualité agronomique est un obstacle pour les cultures irriguées. Mais, conséquence d'une surcharge pastorale, ce milieu est marqué par le surpâturage et le déboisement intensif, causes de déstabilisation des sols.

La problématique de l'irrigation, et plus généralement de l'accès et de l'utilisation des ressources en eau, est un enjeu fondamental des dynamiques agricoles. Dans la région d'étude, la surface irriguée s'étend sur 2660 ha. L'irrigation est surtout développée dans les grandes exploitations, capables d'investir et de rentabiliser ces infrastructures coûteuses. Cependant, l'absence d'une stratégie collective pour subvenir aux besoins croissants en eau a conduit les exploitants à la mise en irrigation privée de leurs exploitations (Riachi, 2012). En conséquence, des excès de consommation incontrôlés contribuent à l'assèchement des sources et peuvent mener à la limitation du développement du secteur agricole.

\subsection{Les effets récents du conflit syrien : arrivées de réfugiés et conséquences}

Le Liban se trouve mêlé aux développements géopolitiques de la Syrie à plusieurs échelles. La plaine de la Béqaa est impactée par les flux migratoires syriens en lien avec la guerre. En 2015, le Haut-Commissariat des Nations Unies pour les réfugiés y a recensé 24079 familles. Dans le secteur d'étude, le nombre de familles avoisine 350, avec une moyenne de six personnes par ménage, ce qui est supérieur à la taille moyenne des ménages libanais dans la même région (4,7 personnes par ménage). Ces habitats précaires sont installés dans les secteurs agricoles, soit par des familles isolées qui y ont loué des terrains, soit dans des camps de réfugiés construits par des ONG (médecins sans frontières...). 
Ceci ne va pas sans poser divers problèmes sanitaires. L'absence installations d'assainissement amène l'évacuation des eaux usées dans les fossés, ce qui provoque une dégradation de la qualité des eaux souterraines dans le bassin versant du Litani. À cela s'ajoutent les eaux usées des usines (alimentaires, textiles...) limitrophes du Litani. Ces nuisances ont des répercussions sur la production locale et contribuent à l'abandon des terres.

\section{Conclusion}

La méthodologie développée dans le cadre de cette étude a permis de suivre les mutations paysagères à l'aide d'un SIG (Demaze et al., 2002). Selon les différentes dates concernées, les facteurs d'évolution ont été commandés par la conjoncture économique et politique, mais aussi par les conditions d'exploitation et les structures foncières. Parmi les autres éléments décisifs, l'extension de l'urbanisation ou de nouveaux besoins du marché intérieur et international, confortent l'idée que le secteur agricole de la Beqaa Ouest est influencé, comme dans d'autres régions agricoles du monde, par des facteurs extérieurs, tels que la géopolitique des populations ou la forte demande en eau d'irrigation.

En 1950, le secteur agricole contribuait à $20 \%$ du PIB du Liban (Paix, 1975). Aujourd'hui, le budget de l'État attribué à l'agriculture est inférieur à $1 \%$ et la production agricole ne représente que $5 \%$ du PIB. Les acteurs agricoles ont un rôle important dans la valorisation du milieu rural. L'orientation vers des systèmes de production autonomes et économes, qui contribueraient à la baisse des charges en réduisant l'usage des intrants et la consommation en eau, permettraient de développer une agriculture durable (Cousinie, 2010).

Sans doute est-ce le rôle des politiques publiques (réglementation, subventions...) d'œuvrer pour la restauration des surfaces boisées et pour soutenir le secteur agricole, qui souffre de l'absence d'une stratégie à long terme (Lutringer, 2017).

Remerciements. Nous remercions le rédacteur-en-chef et les évaluateurs qui ont accepté de juger ce travail. Nous tenons à remercier de même Ghaleb Faour et Leyla Grizi pour leur collaboration.

\section{Références}

Andrieu J, Mering C. 2008. Cartographie par télédétection des changements de la couverture végétale sur la bande littorale ouestafricaine : exemple des Rivières du Sud du delta du Saloum au Rio Geba. Teledetection 8(2): 93-118. https://halshs.archives-ouvertes. $\mathrm{fr} / \mathrm{halshs}-00388170$.

Arnal C, Laurens L, Soulard C. 2013. Les mutations paysagères engendrées par l'arrachage viticole, un vecteur de mobilisation des acteurs territoriaux dans l'Hérault. Méditerranée 120: 49-58. DOI: 10.4000/mediterranee.6673.

Cousinie P. 2010. Produire autonome et économe sur les territoires l'agriculture durable, une voie d'avenir. ISDA 2010, Jun 2010, Montpellier, France. 11 p. https://hal.archives-ouvertes.fr/hal00525954.
Dan Wenhong D. 2011. Le développement de l'agriculture en milieu karstique dans le Sud-ouest de la Chine : l'exemple du Guizhou (district de Ziyun). Cahiers d'Outre-Mer 1(253-254): 119-134. https://www.cairn.info/revue-les-cahiers-d-outre-mer-2011-1page-119.htm.

Demaze MT, Fotsing JM, Huynh F. 2002. La déforestation dans la région de Saint-Georges de l'Oyapock (Guyane française). Cahiers d'Outre-Mer 55(218): 197-222. https://halshs.archives-ouvertes. fr/halshs-00308968.

Dubertret L, Wetzel R. 1951. Carte géologique au 1/50 000. Feuille de Rachaya et Zebadani au 50000 ${ }^{e}$. Beyrouth: ministère des Travaux Publics. 56 p.

El Hage Hassan H. 2011. Les Apports d'un SIG dans la connaissance des évolutions de l'occupation du sol et de la limitation du risque érosif dans la plaine de la Béqaa (Liban). Exemple d'un secteur du Béqaa el Gharbi. Orléans: Université d'Orléans, thèse de doctorat en géographie, 381 p. https://tel.archives-ouvertes.fr/tel-00647147.

El Hage Hassan H, Touchart L, Faour G. 2013. La sensibilité potentielle du sol à l'érosion hydrique dans l'Ouest de la Béqaa au Liban. Mappemonde 109: 1-17. http://mappemonde.mgm.fr/ num37/articles/art13104.html.

El Hage Hassan H, Touchart L, Ardillier-Carras F, Faour G. 2016. Lutte contre l'érosion et aménagement agricole dans la plaine de la Béqaa (Liban). M@ppmonde 117: 1-17. https://mappemondearchive.mgm.fr/num45/articles/index.html.

El Hage Hassan H, Charbel L, Touchart L. 2018. Modélisation de l'érosion hydrique à l'échelle du bassin versant du Mhaydssé. Béqaa - Liban. VertigO 18(1): 1-19. DOI: 10.4000/vertigo.19804.

FAO. 1989. Évaluation des Terres pour l'Agriculture irriguée. FAO Soils Bulletin 55. http://www.fao.org/3/S8500F/S8500F00.htm.

Foody GM. 2002. Status of land cover classification accuracy assessment. Remote Sensing Environ 80(1): 185-201. DOI: 10.1016/S0034-4257(01)00295-4.

Grégoire C, Litaudon-Jouve C. 2004. Étude de la dynamique de l'occupation du sol en zone viticole AOC sur trois communes du Bas-Rhin. Rev Geogr Est 44(1-2): 43-53. http://rge.revues.org/ 1027.

Hamze M. 1991. L'impact des biotechnologies sur l'agriculture au Liban. CIHEAM-IAMB Options Méditerranéennes 14: 137-140. http://om.ciheam.org/om/pdf/a14/92605124.pdf.

Houet T. 2006. Modélisation prospective de l'occupation du sol en zone agricole intensive dans la France de l'Ouest. Norois 198(1): 35-47. DOI: 10.4000/norois.2025.

Lagabrielle E, Metzger P, Martignac C, Lortic B, Durieux L. 2007. Les dynamiques d'occupation du sol à la Réunion (1989-2002). Mappemonde 86(2): 1-23. https://mappemonde-archive.mgm.fr/ num14/articles/art07205.pdf.

Lutringer C. 2017. Le ciblage des politiques agricoles en Inde : les implications pour les paysans du système d'approvisionnement public. Cahiers Agricultures 26: 45008. DOI: 10.1051/cagri/ 2017027.

Ministère de l'Agriculture du Liban. 1999. Rapport de Synthèse du Recensement Général Agricole.

Ministère de l'Agriculture du Liban. 2008. Enquête sur la production agricole 2006-2007.

Paix C. 1975. La portée spatiale des activités tertiaires de commandement économique au Liban. Rev Tiers Monde 6-61: 135-182. DOI: 10.3406/tiers.1975.2537.

Riachi R. 2012. Agriculture et système alimentaire sous la mission hydraulique libanaise. Méditerranée 119: 35-43. https://journals. openedition.org/mediterranee/6470. 
Sako N, Beltrando G, Atta KF, Didi N'da H, Brou T. 2019. Dynamique forestière et pression urbaine dans le Parc national du Banco (Abidjan, Côte d'Ivoire). VertigO 13(2). DOI: 10.4000/ vertigo.14127.
Skupinski G, Bintan D, Weber C. 2009. Les images satellites Spot multi-dates et la métrique spatiale dans l'étude du changement urbain et suburbain - Le cas de la basse vallée de la Bruche (BasRhin, France). Cybergeo 439: 1-22. DOI: 10.4000/cybergeo.21995.

Citation de l'article : El Hage Hassan H, Ardillier-Carras F, Charbel L. 2019. Les changements d'occupation des sols dans la Béqaa Ouest (Liban): le rôle des actions anthropiques. Cah. Agric. 28: 10. 